\title{
Cryptanalysis of Two Dynamic ID-based Remote User Authentication Schemes for Multi-Server Architecture
}

\author{
Ding Wang ${ }^{1,2 \star}$, Chun-guang Ma ${ }^{1, \star \star}$, De-li Gu${ }^{1}$, and Zhen-shan Cui ${ }^{1}$ \\ 1 Harbin Engineering University, Harbin City 150001, China \\ 2 Automobile Management Institute of PLA, Bengbu City 233011, China \\ wangdingg@mail.nankai.edu.cn; machunguang@hrbeu.edu.cn
}

\begin{abstract}
Understanding security failures of cryptographic protocols is the key to both patching existing protocols and designing future schemes. In NSS'10, Shao and Chin pointed out that Hsiang and Shih's dynamic ID-based remote user authentication scheme for multi-server environment is vulnerable to server spoofing attack and fails to preserve user anonymity, and further proposed an improved version which is claimed to be efficient and secure. In this study, however, we will demonstrate that, although Shao-Chin's scheme possesses many attractive features, it still cannot achieve the claimed security goals, and we report its following flaws: (1) It cannot withstand offline password guessing attack under their non-tamper resistance assumption of the smart card; (2) It fails to provide user anonymity; (3) It is prone to user impersonation attack. More recently, Li et al. found that Sood et al.'s dynamic ID-based authentication protocol for multi-server architecture is still vulnerable to several kinds of attacks and presented a new scheme that attempts to overcome the identified weaknesses. Notwithstanding their intentions, Li et al.'s scheme is still found vulnerable to various known attacks by researchers. In this study, we perform a further cryptanalysis and uncover its two other vulnerabilities: (1) It cannot achieve user anonymity, the essential goal of a dynamic ID-based scheme; (2) It is susceptible to offline password guessing attack. The proposed cryptanalysis discourages any use of the two schemes under investigation in practice and reveals some subtleties and challenges in designing this type of schemes.
\end{abstract}

Keywords: Cryptanalysis, Authentication protocol, Offline password guessing attack, Smart card, Multi-Server

\section{Introduction}

With the rapid growth of Internet applications, the number of service providing servers proliferates at an ever-increasing rate $[1,2]$. The distributed locations

* Corresponding author.

** The abridged version of this paper is going to appear in the proceedings of NSS 2012, Lecture Notes in Computer Science, Springer-Verlag. 
of service servers make it convenient and efficient for subscribers to access resources, and it is of great concern to protect the users and systems' security and privacy from malicious adversaries. Accordingly, user authentication is crucial to assure one communicating party of the legitimacy of the corresponding party by acquisition of corroborative evidence, preventing unauthorized clients from accessing system services for multi-server environment. Among numerous methods for user authentication, password based authentication using smart cards is the most convenient and effective two-factor authentication mechanism and has been widely adopted in many security-critical applications, such as e-banking, e-commerce and e-health [3].

In 1991, Chang and Wu [4] introduced the first password based remote user authentication schemes using smart cards, since then there have been many of this type of schemes proposed [5-12]. Although the issue of password authentication with smart cards for single-server environment recently has already been well studied [9-12], it is extremely difficult for a user to remember these numerous different sets of identities and passwords when he/she employs these single-server authentication schemes to login and access different remote service servers.

To address this issue, a number of smart card based password authentication schemes for multi-server environment has been presented quite recently [1316]. A sound and practical remote user authentication protocol for multi-server environment should be of high efficiency and can resist various related attacks, as well as the provision of some desirable features, such as mutual authentication, key agreement, local password update, user anonymity and so on. However, all of these schemes for multi-server environment are found impractical or completely insecure shortly after they were first proposed [17-19], which outlines the need for intensive further research and dynamic ID-based schemes that can preserve user anonymity are of particular interest.

In 2010, Shao and Chin [20] proposed an improved dynamic ID-based authentication scheme for multi-server environment to overcome the weakness of Hsiang-Shin's scheme [16]. The authors claimed that their improvement provides mutual authentication and is free from all related cryptographic attacks, such as replay attack, offline password guessing attack, insider attack, impersonation attack and so on. Although their scheme is efficient and superior to the previous solutions for implementation in resource-constrained applications, e.g. mobile devices, we find their scheme cannot achieve the claimed security: their scheme is vulnerable to offline password attack and user impersonation attack, and fails to preserve user anonymity.

More recently, Li et al. [21] pointed out that, besides a design flaw, Sood et al. scheme [19] is susceptible to leak-of-verifier attack and stolen smart card attack, and further proposed an efficient and secure dynamic ID-based authentication scheme using smart cards for multi-server architecture to cope with these identified problems. Unfortunately, just two months after Li et al.'s scheme was first published online, the replay attack, password guessing attack and masquerade attack are identified in their scheme by Han [22]. Later on, Xue et al. [23] al- 
so found Li et al.'s scheme cannot withstand the replay attack, denial of service attack, eavesdropping attack, internal attack and impersonation attack. Surprisingly, our further cryptanalysis demonstrates that Li et al.'s scheme still cannot preserve user anonymity, which is the most essential goal of a dynamic ID-based scheme. Besides, we also observed that Li et al.'s scheme is susceptible to another type of offline password guessing attack, which is more effective than and different from Han's. In addition, we point out that Xue et al.'s improvement over Li et al.'s scheme is still vulnerable to a similar offline password guessing attack.

The remainder of this paper is organized as follows: in Section 2, we review Shao-Chin's scheme. Section 3 describes the weaknesses of Shao-Chin's scheme. $\mathrm{Li}$ et al.'s scheme is reviewed in Section 4 and the corresponding cryptanalysis is given is Section 5. Section 6 concludes the paper.

\section{Review of Shao-Chin's scheme}

In this section, we examine the dynamic ID-based authentication scheme using smart cards proposed by Shao and Chin [20] in NSS 2010. Shao-Chin's scheme consists of five phases: registration phase, login phase, authentication phase, password change phase and track phase. For ease of presentation, we employ some intuitive abbreviations and notations listed in Table 1 and we will follow the notations in Shao-Chin's scheme as closely as possible.

Table 1. Notations

\begin{tabular}{ll}
\hline Symbol & Description \\
\hline$U_{i}$ & $i^{t h}$ user \\
$S$ & remote server \\
$I D_{i}$ & identity of user $U_{i}$ \\
$C I D_{i}$ & dynamic identity of user $U_{i}$ \\
$P_{i}$ & password of user $U_{i}$ \\
$S_{j}$ & $j^{\text {th }}$ service providing server \\
$S I D_{j}$ & identity of service server $S_{j}$ \\
$\oplus$ & the bitwise XOR operation \\
$\|$ & the string concatenation operation \\
$h(\cdot)$ & collision free one-way hash function \\
$A \rightarrow B: M$ & message $M$ is transferred through a common channel from $A$ to $B$ \\
$A \Rightarrow B: M$ & message $M$ is transferred through a secure channel from $A$ to $B$ \\
\hline
\end{tabular}

Besides the users and the service servers, there is another participant, called registration center $(R C)$, involving in the system, and $R C$ is trusted by all the users and service servers. Let $x$ and $z$ be two secret keys of $R C$.

\subsection{Registration phase}

The registration phase is divided into two parts, namely, the server registration and the user registration. 
(i) Server registration

1) $S_{j}$ chooses his/her identity $S I D_{j}$;

2) $S_{j} \Rightarrow R C:\left\{S I D_{j}\right\}$;

3) $R C$ computes $y_{j}=h\left(h(x) \| S I D_{j}\right)$ and $h(z)$;

4) $R C \Rightarrow S_{j}:\left\{y_{j}, h(z)\right\}$.

(ii) User registration

1) $U_{i}$ chooses his/her $I D_{i}$ and $P_{i}$

2) $U_{i} \Rightarrow R C:\left\{I D_{i}, P_{i}\right\}$

3) $R C$ computes $T_{i}=h\left(I D_{i} \| x\right), R_{i}=h(x) \oplus h(z) \oplus T_{i}, V_{i}=T_{i} \oplus h\left(I D_{i} \|\right.$

$\left.P_{i}\right)$ and $H_{i}=h\left(T_{i}\right)$ and stores $\left\{R_{i}, V_{i}, H_{i}, h(\cdot)\right\}$ in the smart card;

4) $R C \Rightarrow U_{i}$ : A smart card containing security parameters $\left\{R_{i}, V_{i}, H_{i}, h(\cdot)\right\}$.

\subsection{Login phase}

When $U_{i}$ wants to login to $S_{j}$, the following operations will be performed:

Step L1. $U_{i}$ inserts her smart card into card reader, and inputs $I D_{i}$ and $P_{i}$.

Step L2. Smart card computes $T_{i}=V_{i} \oplus h\left(I D_{i} \| P_{i}\right)$, and checks whether $H_{i}$ equals $h\left(T_{i}\right)$ or not. If they are equal, the user proceeds to the next step. Otherwise, the login request is rejected.

Step L3. Smart card generates a random number $r$ and computes $B_{1}=R_{i} \oplus$ $T_{i} \oplus h\left(r \| T_{i}\right)$

Step L4. $U_{i} \rightarrow S_{j}:\left\{B_{1}\right\}$.

Step L5. On receiving $B_{1}$ from $U_{i}, S_{j}$ computes $B_{2}=B_{1} \oplus h(z)$;

Step L6. $S_{j} \rightarrow U_{i}:\left\{B_{2}\right\}$.

Step L7. Smart card chooses a random number $N_{i}$ and computes $y_{j}=h\left(B_{2} \oplus\right.$ $\left.h\left(r \| T_{i}\right) \| S I D_{j}\right), C I D_{i}=I D_{i} \oplus h\left(B_{2} \oplus h\left(r \| T_{i}\right) \| N_{i}\right), G_{i}=C I D_{i} \oplus$ $h\left(y_{j} \| N_{i}\right)$ and $C=h\left(C I D_{i}\left\|G_{i}\right\| N_{i}\right)$.

Step L8. $U_{i} \rightarrow S_{j}:\left\{C, G_{i}, N_{i}\right\}$

\subsection{Authentication phase}

After receiving the login request from $U_{i}, S_{j}$ performs the following operations:

Step A1. The server $S_{j}$ Computes $C I D_{i}=G_{i} \oplus h\left(y_{j} \| N_{i}\right)$ and, then checks whether the received $C$ is equal to the computed $h\left(C I D_{i}\left\|G_{i}\right\| N_{i}\right)$. If the equality does not hold, the server $S_{j}$ rejects the login request.

Step A2. $S_{j}$ generates a random number $N_{j}$ and computes $M_{1}=h(C I D \|$ $\left.S I D_{j} \| N_{i}\right)$.

Step A3. $S_{j} \rightarrow U_{i}:\left\{M_{1}, N_{j}\right\}$.

Step A4. Upon receiving the response message from $S_{j}, U_{i}$ computes $h\left(C I D_{i} \|\right.$ $\left.S I D_{j} \| N_{j}\right)$ and compares it with $M_{1}$. The equality indicates the legitimacy of $S_{j}$. Otherwise, the login request is interrupted.

Step A5. $U_{i}$ computes $M_{2}=h\left(C I D_{i}\left\|S I D_{j}\right\| N_{j}\right)$.

Step A6. $U_{i} \rightarrow S_{j}:\left\{M_{2}\right\}$. 
Step A7. On receiving $M_{2}, S_{j}$ checks whether the received $M_{2}$ equals the computed $h\left(C I D_{i}\left\|S I D_{j}\right\| N_{j}\right)$. The equality indicates the legitimacy of $U_{i}$. Otherwise, the access request is interrupted.

Step A8. After authenticating each other, $U_{i}$ and $S_{j}$ use the same session key $S K=h\left(C I D_{i}\left\|S I D_{j}\right\| N_{i} \| N_{j}\right)$ to secure subsequent data communications.

\subsection{Password change phase and track phase}

Since both the password change phase and track phase have little relevance with our discussions, they are omitted here.

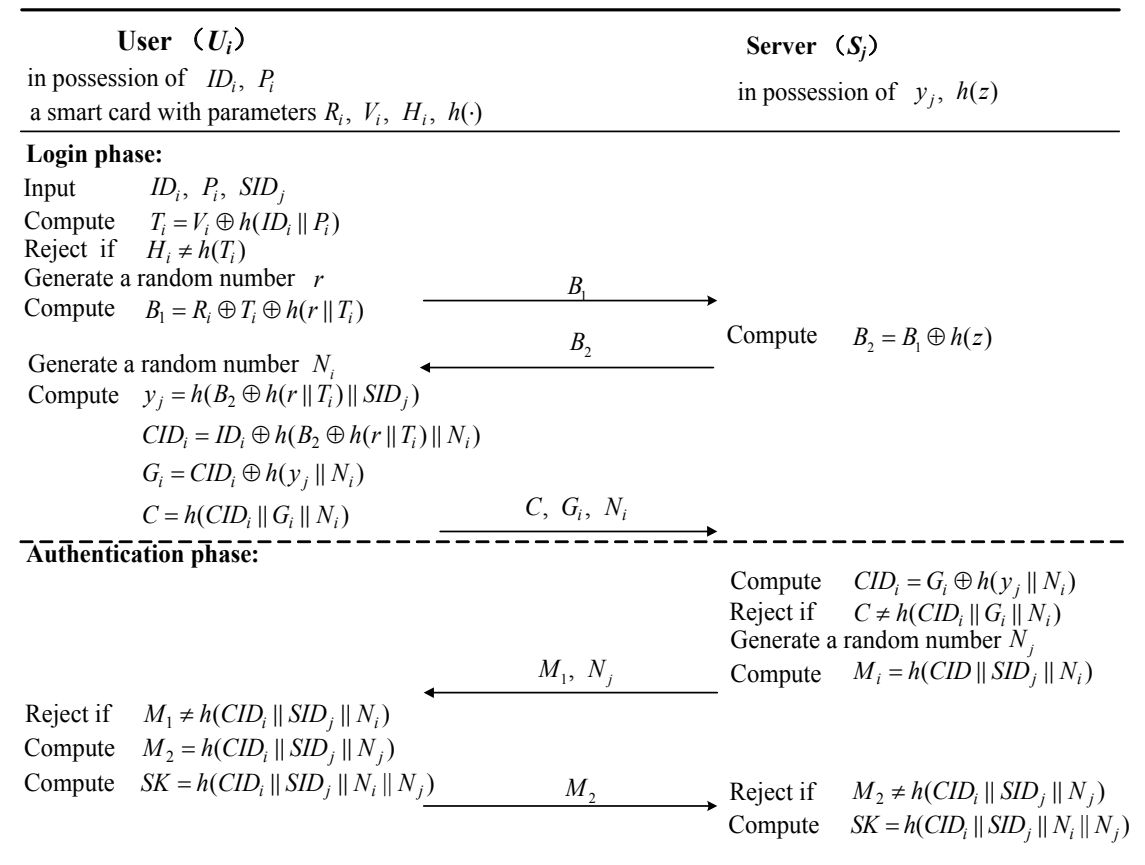

Fig. 1. Login and authentication phase of Shao-Chin's scheme

\section{Cryptanalysis of Shao-Chin's scheme}

There are three assumptions explicitly made in Shao-Chin's scheme [20]:

(i) An adversary $\mathcal{A}$ has total control over the communication channel between the user $U$ and the remote server $S$. In other words, the attacker can insert, alter, delete or intercept any messages exchanged in the channel. 
(ii) The secret parameters stored in the smart card can be revealed once a legitimate user's smart card is somehow obtained (e.g. picked up or stolen) by $\mathcal{A}$.

(iii) The passwords are weak, i.e. of low entropy.

Note that the above three assumptions, which are also made in the latest works [9-12,17-19], are indeed reasonable: (1) Assumption $i$ is accordant with the common Dolev-Yao adversary model for distributed communication; (2) Assumption $i i$ is practical when taking the state-of-the-art side-channel attack techniques [24-26] into consideration; and (3) Assumption iii reveals the reality that a user is allowed to choose her own password at will during the password change phase and registration phase, usually the user is apt to select a password which is easily remembered for her convenience [27], e.g. her birthday or home phone number, and the human-memorable password tends to be "weak password" [28].

In the following discussions of the security pitfalls of Shao-Chin's scheme, based on the above three assumptions, we assume that an adversary can extract the secret parameters $\left\{V_{i}, R_{i}, H_{i}\right\}$ stored in the legitimate user's smart card, and could also intercept or block the exchanged messages $\left\{B_{1}, B_{2}, C, G_{i}, N_{i}, M_{i}, N_{j}, M_{2}\right\}$ during the login and authentication phase.

\subsection{No provision of user anonymity}

A protocol preserving user anonymity prevents an adversary from acquiring sensitive information about an individual's social circle, preferences, lifestyles, shopping patterns, etc. by analyzing the login history, the services requested, or the communications being accessed [29]. In addition, the leakage of user-specific information may cause an unauthorized entity or malicious attacker to track the user's current location and login history [30]. Hence, assuring anonymity not only does protect user privacy but also makes remote user authentication protocols more secure. In Shao-Chin's scheme, the dynamic-ID technique is employed to provide the feature of user anonymity, however, the following attack demonstrates the failure of their attempt.

Let us see how a dishonest service provider $S_{k}$ colluding with a malicious privileged user $U_{m}$ successfully breach the anonymity of any legitimate user, say $U_{i} . U_{m}$ having her own smart card can gather information $R_{m}, V_{m}$ from her own smart card, with previously intercepted authentication messages $\left\{B_{1}, B_{2}, N_{i}, G_{i}, C\right\}$ that are exchanged between $U_{m}$ and any service provider, say $S_{j}, U_{m}$ and $S_{k}$ can collude to compute $I D_{i}$ corresponding to $U_{i}$ as follows:

Step 1. $U_{m}$ computes $T_{m}=V_{m} \oplus h\left(I D_{m} \| P_{m}\right)$, as $V_{m}$ is revealed from her own smart card, $I D_{m}$ and $P_{m}$ is known to herself;

Step 2. $U_{m}$ computes $h(x) \oplus h(z)=R_{m} \oplus T_{m}$, where $R_{m}$ is revealed;

Step 3. $U_{m}$ and $S_{k}$ collude to compute $h(x)=(h(x) \oplus h(z)) \oplus h(z)=\left(R_{m} \oplus\right.$ $\left.T_{m}\right) \oplus h(z)$, where $h(z)$ is known to all service servers, including $S_{k}$.

Step 4. Guesses $U_{i}$ 's identity to $I D_{i}^{*}$; 
Step 5. Computes $C I D_{i}^{*}=I D_{i}^{*} \oplus h\left(h(x) \| N_{i}\right)$, as

$$
\begin{aligned}
h(x) & =h(x) \oplus\left(h\left(r \| T_{i}\right) \oplus h\left(r \| T_{i}\right)\right) \oplus(h(z) \oplus h(z)) \\
& =\left(h(x) \oplus h(z) \oplus h\left(r \| T_{i}\right)\right) \oplus\left(h(z) \oplus h\left(r \| T_{i}\right)\right) \\
& =B_{1} \oplus\left(h(z) \oplus h\left(r \| T_{i}\right)\right) \\
& =B_{2} \oplus h\left(r \| T_{i}\right) .
\end{aligned}
$$

Step 6. Computes $C^{*}=h\left(C I D_{i}^{*}\left\|G_{i}\right\| N_{i}\right)$, where $G_{i}$ and $N_{i}$ is intercepted.

Step 7. Verifies the correctness of $I D_{i}^{*}$ by checking if the computed $C^{*}$ is equal to the intercepted $C$;

Step 8. Goes back to Step 4 until the correct value of $I D_{i}$ is found.

In practice, a user's identity is often drawn from a very limited space, say $\mathcal{D}_{i d}$, the above procedure can be completed in polynomial time.

It is worth noting that, in the above attack, the malicious user $U_{m}$ only needs to extract the security parameters stored in her own smart card, she does not need to obtain any information about the victim user $U_{i}$ except the public authentication messages originating from $U_{i}$. As a result, the above attack is effective and practical. In conclusion, once an internal user colludes with a dishonest service server, user anonymity will be breached in Shao-Chin's scheme, while user anonymity is the most essential security feature that a dynamic identity-based authentication scheme is designed to provide.

\subsection{Offline password guessing attack}

As stated in Section 3.1, any legitimate user $U_{i}$ 's identity can be breached when an internal malicious user $U_{m}$ colludes with a service server $S_{k}$. Once the victim user $U_{i}$ 's identity $I D_{i}$ is obtained by $U_{m}$ and $S_{k}, U_{i}$ 's password $P_{i}$ can also be offline guessed as follows:

Step 1. Guesses the value of $P_{i}$ to be $P_{i}^{*}$ from a dictionary space $\mathcal{D}_{p w}$.

Step 2. Computes $T_{i}^{*}=h\left(I D_{i} \| P_{i}^{*}\right) \oplus V_{i}$, where $V_{i}$ is extracted from $U_{i}$ 's smart card.

Step 3. Verifies the correctness of $P_{i}^{*}$ by checking if the computed $h\left(T_{i}^{*}\right)$ is equal to the revealed $H_{i}$

Step 4. Repeats the above steps until the correct value of $P_{i}$ is found.

Let $\left|\mathcal{D}_{i d}\right|$ and $\left|\mathcal{D}_{p w}\right|$ denote the number of identities in identity space $\mathcal{D}_{i d}$ and the number of passwords in password space $\mathcal{D}_{p w}$, respectively. The running time of the above attack procedure is $\mathcal{O}\left(\left|\mathcal{D}_{i d}\right| *\left(3 T_{H}+5 T_{X}\right)+\left|\mathcal{D}_{p w}\right| *\left(2 T_{H}+T_{X}\right)\right)$, where $T_{H}$ is the running time for Hash operation and $T_{X}$ is the running time for $X O R$ operation. Since both password and identity are human-memorable short strings but not high-entropy keys, in other words, they are often chosen from two corresponding dictionaries of small size, e.g. $\left|\mathcal{D}_{i d}\right| \leq\left|\mathcal{D}_{p w}\right|=10^{6}[28]$. As $\left|\mathcal{D}_{i d}\right|$ and $\left|\mathcal{D}_{p w}\right|$ are very limited in practice, the above attack can be completed in polynomial time.

Note that, in this attack, the malicious user $U_{m}$ not only needs to extract the security parameters stored in her own smart card, but also needs to obtain the secret information stored in the smart card of victim user $U_{i}$. Although 
this assumption is much constrained, our attack demonstrates the feasibility of offline password guessing attack on Shao-Chin's scheme under their non-tamper resistance assumption of the smart card, thereby contradicting the claim made in $[20]$.

\subsection{User impersonation attack}

An internal malicious user $U_{m}$ and a service server $S_{k}$ can collude to impersonate any legitimate user (even non-existent user), say $U_{\text {ran }}$, to login any service server, say $S_{j}$, as follows:

Step 1. $U_{m}$ computes $T_{m}=V_{m} \oplus h\left(I D_{m} \| P_{m}\right)$, where $V_{m}$ is revealed from her own smart card, $I D_{m}$ and $P_{m}$ is known to herself;

Step 2. $U_{m}$ computes $h(x) \oplus h(z)=R_{m} \oplus T_{m}$, where $R_{m}$ is revealed;

Step 3. $S_{k}$ and $U_{m}$ collude to compute $h(x)=(h(x) \oplus h(z)) \oplus h(z)=\left(R_{m} \oplus\right.$ $\left.T_{m}\right) \oplus h(z)$, where $h(z)$ is known to all service servers, including $S_{k}$.

Step 4. $U_{m}$ sends a random value $X$ to any service server, say $S_{j}$;

Step 5. $U_{m}$ ignores the response $\left\{B_{2}\right\}$ sent back by $S_{j}$ and computes $y_{j}=$ $h\left(h(x) \| S I D_{j}\right)$, where $S I D_{j}$ is $S_{j}$ 's identity.

Step 6. $U_{m}$ computes $C I D_{\text {ran }}=I D_{\text {ran }} \oplus h\left(h(x) \| N_{\text {ran }}\right), G_{\text {ran }}=C I D_{\text {ran }} \oplus$ $h\left(y_{j} \| N_{\text {ran }}\right)$ and $C=h\left(C I D_{\text {ran }}\left\|G_{\text {ran }}\right\| N_{\text {ran }}\right)$, where $N_{\text {ran }}$ is a random number chosen by $U_{m}$.

Step 7. $U_{m}$ sends $\left\{C, G_{\text {ran }}, N_{\text {ran }}\right\}$ to $S_{j}$.

Step 8. On receiving the response $\left\{M_{\text {ran }}, N_{j}\right\}$ sent back by $S_{j}, U_{m}$ computes $M_{2}=h\left(C I D_{\text {ran }}\left\|S I D_{j}\right\| N_{j}\right)$ and the session key $S K=$ $h\left(C I D_{\text {ran }}\left\|S I D_{j}\right\| N_{\text {ran }} \| N_{j}\right)$.

Step 9. $U_{m}$ sends $\left\{M_{2}\right\}$ to $S_{j}$.

It is easy to see that: 1$)$ On receiving $X$ sent by $U_{m}$ in Step $4, S_{j}$ will send back $B_{2}=X \oplus h(z)$ according to the protocol; 2) On receiving $\left\{C, G_{\text {ran }}, N_{\text {ran }}\right\}$ sent by $U_{m}$ in Step $7, S_{j}$ will find no abnormality when checking the validity of $C$, because $U_{m}$ indeed has computed the correct $y_{j}=h\left(h(x) \| S I D_{j}\right)=h\left(B_{2} \oplus\right.$ $\left.h\left(r \| T_{i}\right) \| S I D_{j}\right)$ in Step 5 as

$$
\begin{aligned}
h(x) & =h(x) \oplus\left(h\left(r \| T_{i}\right) \oplus h\left(r \| T_{i}\right)\right) \oplus(h(z) \oplus h(z)) \\
& =\left(h(x) \oplus h(z) \oplus h\left(r \| T_{i}\right)\right) \oplus\left(h(z) \oplus h\left(r \| T_{i}\right)\right) \\
& =B_{1} \oplus\left(h(z) \oplus h\left(r \| T_{i}\right)\right) \\
& =B_{2} \oplus h\left(r \| T_{i}\right) .
\end{aligned}
$$

3) On receiving $M_{2}$ sent by $U_{m}$ in Step $9, S_{j}$ will find no abnormality when checking the validity of $M_{2}$, because $U_{m}$ has indeed computed the valid $C I D_{\text {ran }}=$ $I D_{\text {ran }} \oplus h\left(h(x) \| N_{\text {ran }}\right)$ in Step 5 where $h(x)=B_{2} \oplus h\left(r \| T_{i}\right)$.

It should be noted that, as with the password guessing attack presented in Section 3.1, in this attack, the malicious user $U_{m}$ only needs to extract the security parameters stored in her own smart card, she does not need to obtain any information about the victim user $U_{i}$ except the public authentication messages originating from $U_{i}$. As a result, this impersonation attack is effective and practical. 


\section{Review of Li et al.'s scheme}

In this section, we briefly review the dynamic identity based authentication protocol for multi-server architecture using smart cards proposed by $\mathrm{Li}$ et al. in 2012. Li et al.'s protocol also involves three participants, i.e., the user $\left(U_{i}\right)$, the service providing server $\left(S_{j}\right)$ and the control server $(C S)$. It is should be noted that $C S$, a trusted party, is not only responsible for the registration but also involved in the authentication process of $U_{i}$ and $S_{j} . C S$ is in possession of a master secret key $x$ and a secret number $y$. There are four phases in their protocol: registration, login, authentication and session key agreement, and password change. In the following, we employ the notations listed in Table 1.

\subsection{Registration phase}

The registration phase can be divided into two parts, namely, the server registration and the user registration.

(i) Server registration

1) $S_{j}$ chooses his/her identity $S I D_{j}$;

2) $S_{j} \Rightarrow C S:\left\{S I D_{j}\right\}$;

3) $C S$ computes $h\left(S I D_{j} \| y\right)$ and $h(x \| y)$;

4) $C S \Rightarrow S_{j}:\left\{h(x \| y), h\left(S I D_{j} \| y\right)\right\}$.

(ii) User registration

1) $U_{i}$ freely chooses his/her $I D_{i}$ and $P_{i}$, and chooses a random number $b$. Then, $U_{i}$ computes $A_{i}=h\left(b \| P_{i}\right)$;

2) $U_{i} \Rightarrow C S:\left\{I D_{i}, A_{i}\right\}$;

3) $C S$ computes $B_{i}=h\left(I D_{i} \| x\right), C_{i}=h\left(I D_{i}\|h(y)\| A_{i}\right), D_{i}=$ $B_{i} \oplus h\left(I D_{i} \| A_{i}\right), E_{i}=B_{i} \oplus h(y \| x)$, and stores $\left\{C_{i}, D_{i}, E_{i}, h(\cdot), h(y)\right\}$ in the smart card;

4) $C S \Rightarrow U_{i}$ : A smart card containing parameters $\left\{C_{i}, D_{i}, E_{i}, h(\cdot), h(y)\right\}$.

5) Upon receiving the smart card, $U_{i}$ enters $b$ into it.

\subsection{Login phase}

When $U_{i}$ wants to login to $S_{j}$, the following operations will be performed:

Step L1. User $U_{i}$ inserts her smart card into a card reader and inputs her identity $I D_{i}$, password $P_{i}$ and the service server's identity $S I D_{j}$.

Step L2. The smart card computes $A_{i}=h\left(b \| P_{i}\right)$ and $C_{i}^{\prime}=h\left(I D_{i}\|h(y)\| A_{i}\right)$, and checks whether $C_{i}^{\prime}=C_{i}$. If they are equal, it indicates that $U_{i}$ is a legal card holder.

Step L3. The smart card generates a random number $N_{i 1}$, and computes $B_{i}=$ $D_{i} \oplus h\left(I D_{i} \| A_{i}\right), F_{i}=h(y) \oplus N_{i 1}, P_{i j}=E_{i} \oplus h\left(h(y)\left\|N_{i 1}\right\| S I D_{j}\right)$, $C I D_{i}=A_{i} \oplus h\left(B_{i}\left\|F_{i}\right\| N_{i 1}\right), G_{i}=h\left(B_{i}\left\|A_{i}\right\| N_{i 1}\right)$.

Step L4. $U_{i} \rightarrow S_{j}:\left\{F_{i}, G_{i}, P_{i j}, C I D_{i}\right\}$. 


\subsection{Authentication and session key agreement phase}

Step A1. On receiving the login request, $S_{j}$ chooses a random number $N_{i 2}$, and computes $K_{i}=h\left(S I D_{j} \| y\right) \oplus N_{i 1}$ and $M_{i}=h\left(h(x \| y) \| N_{i 2}\right)$.

Step A2. $S_{j} \rightarrow C S:\left\{F_{i}, G_{i}, P_{i j}, C I D_{i}, S I D_{j}, K_{i}, M_{i}\right\}$.

Step A3. Upon receiving the login request $\left\{F_{i}, G_{i}, P_{i j}, C I D_{i}, S I D_{j}, K_{I}, M_{i}\right\}$, $C S$ computes $N_{i 2}=K_{i} \oplus h\left(S I D_{j} \| y\right), M_{i}^{\prime}=h\left(h(x \| y) \| N_{i 2}\right)$, and checks whether the computed $M_{i}^{\prime}$ equals the received $M_{i}$. If they are equal, the validity of the server $S_{j}$ is verified by the control server $C S$. Otherwise, the $C S$ terminates the session.

Step A4. $C S$ computes $N_{i 1}=F_{i} \oplus h(y), B_{i}=P_{i j} \oplus h\left(h(y)\left\|N_{i 1}\right\| S I D_{j}\right) \oplus h(y \| x)$ $\left(=E_{i} \oplus h(y \| x)\right), A_{i}=C I D_{i} \oplus h\left(B_{i}\left\|F_{i}\right\| N_{i 1}\right), G_{i}^{\prime}=h\left(B_{i}\left\|A_{i}\right\| N_{i 1}\right)$ and checks $G_{i}^{\prime} \stackrel{?}{=} G_{i}$. If the verification holds, the legitimacy of user $U_{i}$ is authenticated by $C S$. Otherwise $C S$ terminates the session.

Step A5. The control server $C S$ generates a random number $N_{i 3}$, and computes $Q_{i}=N_{i 1} \oplus N_{i 3} \oplus h\left(S I D_{j} \| N_{i 2}\right), R_{i}=h\left(A_{i} \| B_{i}\right) \oplus h\left(N_{i 1} \oplus N_{i 2} \oplus N_{i 3}\right)$, $V_{i}=h\left(h\left(A_{i} \| B_{i}\right) \| h\left(N_{i 1} \oplus N_{i 2} \oplus N_{i 3}\right)\right), T_{i}=N_{i 2} \oplus N_{i 3} \oplus h\left(A_{i} \|\right.$ $\left.B_{i} \| N_{i 1}\right)$.

Step A6. $C S \rightarrow S_{j}:\left\{Q_{i}, R_{i}, V_{i}, T_{i}\right\}$.

Step A7. On receiving the authentication message $\left\{Q_{i}, R_{i}, V_{i}, T_{i}\right\}$ from $C S$, server $S_{j}$ computes $N_{i 1} \oplus N_{i 3}=Q_{i} \oplus h\left(S I D_{j} \| N_{i 2}\right), h\left(A_{i} \| B_{i}\right)=$ $R_{i} \oplus h\left(N_{i 1} \oplus N_{i 3} \oplus N_{i 2}\right), V_{i}^{\prime}=h\left(h\left(A_{i} \| B_{i}\right) \| h\left(N_{i 1} \oplus N_{i 3} \oplus N_{i 2}\right)\right)$, and checks $V_{i}^{\prime} \stackrel{?}{=} V_{i}$. If they are not equal, $S_{j}$ terminates the session. Otherwise, the legitimacy of $C S$ is authenticated by the server $S_{j}$.

Step A8. $S_{j} \rightarrow U_{i}:\left\{V_{i}, T_{i}\right\}$.

Step A9. Upon receiving $\left\{V_{i}, T_{i}\right\}$ from $S_{j}$, the smart card computes $N_{i 2} \oplus N_{i 3}=$ $T_{i} \oplus h\left(A_{i}\left\|B_{i}\right\| N_{i 1}\right), V_{i}^{\prime}=h\left(h\left(A_{i} \| B_{i}\right) \| h\left(N_{i 2} \oplus N_{i 3} \oplus N_{i 1}\right)\right)$, and checks $V_{i}^{\prime} \stackrel{?}{=} V_{i}$. If the verification fails, the user $U_{i}$ terminates the session. Otherwise, the legitimacy of the control server $C S$ and the server $S_{j}$ is authenticated by user $U_{i}$.

Finally, the user $U_{i}$, the server $S_{j}$ and the control server $C S$ agree on a common session key $S K=h\left(h\left(A_{i} \| B_{i}\right) \|\left(N_{i 1} \oplus N_{i 2} \oplus N_{i 3}\right)\right)$.

\subsection{Password change phase}

This phase is performed locally. When the user wants to update her password, this phase is invoked. Since this phase has little relevance with our discussions, it is omitted here.

\section{Cryptanalysis of $\mathrm{Li}$ et al.'s scheme}

The three assumptions presented in Section 3 are also explicitly made in Li et al.'s paper when they analyze the security of Sood et al.'s scheme, and thus our following cryptanalysis is also based on these three assumptions. 
Although Li et al.'s scheme has many attractive properties, such as provision of local password change, high efficiency and no time-synchronization problem, it fails to achieve many of the claimed security goals and has been found vulnerable to replay attack, password guessing attack and user impersonation attack by Han [22]. Besides these security pitfalls, later on Xue et al. further found it prone to leak-of-verifier attack, server spoofing attack and denial of service attack, ${ }^{1}$ and they also presented an improvement.

Surprisingly, our further cryptanalysis demonstrates that Li et al.'s scheme still cannot preserve user anonymity, which is the most crucial goal of a dynamic ID-based scheme. Besides, we also observe that Li et al.'s scheme is susceptible to another type of offline password guessing attack, which is more effective than and different from Han's. Furthermore, we point out that Xue et al.'s improvement over Li et al.'s scheme is still vulnerable to a similar offline password guessing attack.

\subsection{No provision of user anonymity}

Let us see how a dishonest service provider $S_{k}$ colluding with a malicious internal user $U_{m}$ successfully breach the anonymity of any legitimate user, say $U_{i} . U_{m}$ having her own smart card can gather information $h(y)$ from her own smart card, with previously intercepted authentication messages $\left\{P_{i j}, S I D_{j}\right\}$ that are exchanged between $U_{m}, C S$ and any service provider, say $S_{j}, U_{m}$ and $S_{k}$ can collude to compute $E_{i}$ corresponding to any user $U_{i}$ as follows:

Step 1. $U_{m}$ extracts $h(y)$ from her own smart card;

Step 2. $U_{m}$ and $S_{k}$ collude to compute $N_{i 1}=F_{i} \oplus h(y)$, where $F_{i}$ is intercepted from the public channel;

Step 3. $U_{m}$ and $S_{k}$ collude to compute $E_{i}=P_{i j} \oplus h\left(h(y)\left\|N_{i 1}\right\| S I D_{j}\right)$, where $P_{i j}$ and $S I D_{j}$ are intercepted from the public channel.

As $E_{i}$ is kept the same for all the login requests of user $U_{i}$ and is specific to user $U_{i}$, this $E_{i}$ can be seen as user $U_{i}$ 's identification. And an adversary can, therefore, use this information to identify and trace $U_{i}$ 's login requests and activities. By generalizing the above attack, any legal user who logins to service servers would be exposed to $U_{m}$ and $S_{k}$, and thus the scheme fails to achieve user anonymity.

It should be noted that, in the above attack, the malicious user $U_{m}$ only needs to extract the security parameters stored in her own smart card, she does not need to obtain any information about the victim user $U_{i}$ except the public authentication messages originating from $U_{i}$. As a result, the above attack is effective and practical. In conclusion, once an internal user colludes with a dishonest service server, user anonymity will be breached in Li et al.'s scheme, while user anonymity is the most crucial security feature that a dynamic identitybased authentication scheme is designed to provide.

${ }^{1}$ We think Xue et al.'s internal attack and eavesdropping attack only constitute parts of replay attack, server spoofing attack, etc, and they may not be considered as independent kinds of attacks, and thus they are not listed here. 


\subsection{Offline password guessing attack}

Let us consider the following scenarios. In case a legitimate user $U_{i}$ 's smart card is stolen by a malicious internal user $U_{m}$, and the stored secret values $h(y), D_{i}, E_{i}$ and $b$ can be extracted. Note that this assumption is reasonable as described in Assumption $i i i$ and also explicitly made in Li et al.'s scheme. With the previously eavesdropped message $\left\{F_{i}, C I D_{i}, G_{i}\right\}$, this malicious internal user $U_{m}$ can successfully guess the password of $U_{i}$ as follows:

Step 1. Extracts $h(y)$ from her own smart card;

Step 2. Computes $N_{i 1}=F_{i} \oplus h(y)$, where $F_{i}$ is intercepted from the public channel;

Step 3. Computes $E_{i}=P_{i j} \oplus h\left(h(y)\left\|N_{i 1}\right\| S I D_{j}\right)$, where $P_{i j}$ and $S I D_{j}$ are intercepted from the public channel.

Step 4. Computes $h(y \| x)=E_{m} \oplus B_{m}=E_{m} \oplus D_{m} \oplus h\left(I D_{m} \| A_{m}\right)=E_{m} \oplus$ $D_{m} \oplus h\left(I D_{m} \| h\left(b \| P_{m}\right)\right)$, where $E_{m}, D_{m}$ and $b$ are revealed from $U_{m}$ 's own smart card;

Step 5. Computes $B_{i}=E_{i} \oplus h(y \| x)$, where $E_{i}$ is revealed from $U_{i}$ 's smart card;

Step 6. Computes $A_{i}=C I D_{i} \oplus h\left(B_{i}\left\|F_{i}\right\| N_{i 1}\right)$;

Step 7. Guesses the value of $P_{i}$ to be $P_{i}^{*}$ from the password space $\mathcal{D}$.

Step 8. Computes $A_{i}^{*}=h\left(b \| P_{i}^{*}\right)$, where $b$ is revealed from $U_{i}$ 's smart card.

Step 9. Verifies the correctness of $P_{i}^{*}$ by checking if $A_{i}^{*}$ equals to $A_{i}$.

Step 10. Repeats Steps 7, 8 and 9 until the correct value of $P_{i}$ is found.

Let $|\mathcal{D}|$ denote the number of passwords in the password space $\mathcal{D}$. Then the running time of the attacker $U_{m}$ is $\mathcal{O}\left(|\mathcal{D}| *\left(5 T_{H}+6 T_{X}\right)\right)$, where $T_{H}$ is the running time for Hash operation and $T_{X}$ is the running time for XOR operation. So, the time for $U_{m}$ to recover the password is a linear function of the number of passwords in the password space. When the password space is small, e.g., $|\mathcal{D}|=10^{6}[28], U_{m}$ may recover the password in seconds on a PC.

It should be noted that, in this attack, the malicious user $U_{m}$ only needs to guess $U_{i}$ 's password, while in the offline password guessing attack proposed by Han [22], the attacker needs to guess both $U_{i}$ 's password and identity correctly at the same time. From this point of view, our attack is more effective. But our disadvantage is that, the adversary in our attack should be an internal user, while the adversary in Han's attack is not subject to this restriction.

\subsection{Offline password guessing attack on Xue et al.'s improvement}

In [23], Xue et al. pointed out that Li et al.'s scheme vulnerable to several attacks and further proposed an improvement that is claimed to be secure. ${ }^{2}$ However, we find Xue et al.'s improvement is still vulnerable to an offline password guessing attack as described in the following.

\footnotetext{
${ }^{2}$ Xue et al.'s improvement has been submitted to Journal of Network and Computer Applications.
} 
Let us consider the following scenarios. In case a legitimate user $U_{i}$ 's smart card is stolen by an adversary $\mathcal{A}$, and the stored secret values such as $C_{i}, D_{i}$ and $b$ can be extracted. Note that this assumption is explicitly made in Xue et al.'s improvement. With a previously eavesdropped message $\left\{F_{i}, P I D_{i}, T S_{i}\right\}, \mathcal{A}$ can acquire $U_{i}$ 's password $P W_{i}$ by performing the following attack procedure:

Step 1. Guesses the value of $P_{i}$ to be $P_{i}^{*}$ from the password space $\mathcal{D}$.

Step 2. Computes $A_{i}^{*}=h\left(b \| P_{i}^{*}\right)$, where $b$ is revealed from $U_{i}$ 's smart card.

Step 3. Computes $B_{i}^{*}=D_{i} \oplus h\left(P I D_{i} \oplus A_{i}^{*}\right)$, where $P I D_{i}$ is intercepted from the public channel.

Step 4. Computes $N_{i 1}^{*}=F_{i} \oplus B_{i}^{*}$.

Step 5. Computes $G_{i}^{*}=b \oplus h\left(B_{i}^{*}\left\|N_{i 1}^{*}\right\| T S_{i} \|\right.$ "11");

Step 6. Verifies the correctness of $P_{i}^{*}$ by checking if $G_{i}^{*}$ equals to the intercepted $G_{i}$.

Step 7. Repeats the above steps until the correct value of $P_{i}$ is found.

Since the size of password dictionary, i.e. $|\mathcal{D}|$, often is very limited in practice, the above attack procedure can be completed in polynomial time.

Notes and Countermeasure. We have analyzed more than sixty recently proposed smart card based password authentication schemes for single-server environment and twelve schemes for multi-server architecture, and find these schemes (no matter for single-server environment or multi-server architecture) that do not employ public-key techniques definitely vulnerable to the offline password guessing attack under the three assumptions (most essentially, the nontamper resistance assumption of the smart card) introduced in Section 3. In other words, all these schemes that do not employ public-key techniques but claim to be secure under these three assumptions are found problematic. A related work done by Halevi and Krawczyk [31] provides very strong evidence (with the probability of $\boldsymbol{P} \neq \boldsymbol{N} \boldsymbol{P}$ ) that, under the common Dolev-Yao adversary model, no password protocol (the traditional one-factor password authentication) can be free from offline password guessing attack if the public-key techniques are not employed. Here, we conjecture that under the three assumptions introduced in Section 3, no smart card based password protocol (two-factor authentication) can be free from offline password guessing attack if the public-key techniques are not employed. And now the countermeasure is obvious: resorting to public-key techniques like [9-12].

\section{Conclusion}

In this paper, we have shown that two dynamic ID-based remote user authentication schemes for multi-server environment are completely broken and only radical revisions of the protocols can possibly eliminate the identified defects and thus the two schemes under investigation are not recommended for practical application. Our results once again demonstrate that no more smart card based password authentication protocols should be constructed with such adhoc methods, and the provable security approach is indispensable for assuring 
a sound protocol. Remarkably, our cryptanalysis highlights the difficulties and challenges in designing secure and efficient dynamic ID-based remote user authentication schemes for multi-server architecture and suggests the need for intensive further research.

Acknowledgment. This research was partially supported by the National Natural Science Foundation of China (NSFC) under Grants No. 61170241 and No. 61073042 , and the open program of State Key Laboratory of Networking and Switching Technology under Grant No. SKLNST-2009-1-10.

\section{References}

1. Bouyoucef, K., Khorasani, K.: A robust distributed congestion-control strategy for differentiated-services network. IEEE Transactions on Industrial Electronics 56(3), 608-617 (2009)

2. Barolli, L., Xhafa, F.: JXTA-OVERLAY: A P2P platform for distributed, collaborative and ubiquitous computing. IEEE Transactions on Industrial Electronics 58(6), 2163-2172 (2010)

3. Lin, S., Hung, M., Tsai, C., Chou, L.: Development of an ease-of-use remote healthcare system architecture using rfid and networking technologies. Journal of Medical Systems pp. 1-15 (2012), doi:10.1007/s10916-012-9836-0

4. Chang, C.C., Wu, T.C.: Remote password authentication with smart cards. IEE Proceedings-Computers and Digital Techniques 138(3), 165-168 (1991)

5. Yang, G.M., Wong, D.S., Wang, H.X., Deng, X.T.: Formal analysis and systematic construction of two-factor authentication scheme. In: Ning, P., Qing, S., Li, N. (eds.) ICICS 2006, LNCS, vol. 4307, pp. 82-91. Springer, Heidelberg (2006)

6. Xu, J., Zhu, W. T., Feng, D. G.: An improved smart card based password authentication scheme with provable security. Computer Standards \& Interfaces 31(4), 723-728 (2009)

7. Yeh, K.H., Su, C.H., Lo, N.W.: Two robust remote user authentication protocols using smart cards. Journal of Systems and Software 83(12), 2556-2565 (2010)

8. Wang, R.C., Juang, W.S., Lei, C.L.: Robust authentication and key agreement scheme preserving the privacy of secret key. Computer Communications 34(3), 274-280 (2011)

9. Ma, C.G., Wang, D., Zhang, Q.M.: Cryptanalysis and improvement of sood et al.s dynamic id-based authentication scheme. In: Ramanujam, R., Ramaswamy, S. (eds.) ICDCIT 2012, LNCS. vol. 7154, pp. 141-152. Springer, Heidelberg (2012)

10. Wu, S.H., Zhu, Y.F., Pu, Q.: Robust smart-cards-based user authentication scheme with user anonymity. Security and Communication Networks 5(2), 236-248 (2012)

11. Wang, D., Ma C.G., Wu, P.: Secure Password-Based Remote User Authentication Scheme with Non-tamper Resistant Smart Cards. In: Cuppens-Boulahia, N., Cuppens, F., Garcia-Alfaro, J. (eds.) (Eds.) DBSec 2012. LNCS, vol. 7371, pp. 114-121, Springer, Heidelberg (2012)

12. Wang, Y. G.: Password protected smart card and memory stick authentication against offline dictionary attacks. In: Gritzalis, D., Furnell, S., Theoharidou, M. (eds.) SEC 2012. IFIP AICT, vol. 376, pp. 489-500. Springer, Boston (2012)

13. Lin, I., Hwang, M., Li, L.: A new remote user authentication scheme for multiserver architecture. Future Generation Computer Systems 19(1), 13-22 (2003) 
14. Tsaur, W., Wu, C., Lee, W.: A smart card-based remote scheme for password authentication in multi-server internet services. Computer Standards \& Interfaces 27(1), 39-51 (2004)

15. Liao, Y., Wang, S.: A secure dynamic id based remote user authentication scheme for multi-server environment. Computer Standards \& Interfaces 31(1), 24-29 (2009)

16. Hsiang, H., Shih, W.: Improvement of the secure dynamic id based remote user authentication scheme for multi-server environment. Computer Standards \& Interfaces 31(6), 1118-1123 (2009)

17. Tan, Z.: Cryptanalysis of two id based password authentication schemes for multiserver environments. International journal of digital content technology and its applications 5(1), 87-94 (2011)

18. Yeh, K., Lo, N., Li, Y.: Cryptanalysis of hsiang-shihs authentication scheme for multi-server architecture. International Journal of Communication Systems 24(7), 829-836 (2011)

19. Sood, S., Sarje, A., Singh, K.: A secure dynamic identity based authentication protocol for multi-server architecture. Journal of Network and Computer Applications 34(2), 609-618 (2011)

20. Shao, M., Chin, Y.: A novel approach to dynamic id-based remote user authentication scheme for multi-server environment. In: 2010 4th International Conference on Network and System Security (NSS 2010). pp. 548-553. IEEE Press, New York (2010)

21. Li, X., Xiong, Y., Ma, J., Wang, W.: An enhanced and security dynamic identity based authentication protocol for multi-server architecture using smart cards. Journal of Network and Computer Applications 35(2), 763-769 (2012)

22. Han, W.: Weaknesses of a dynamic identity based authentication protocol for multiserver architecture. Arxiv preprint arXiv:1201.0883 (2012), http://arxiv.org/ abs/1201.0883

23. Xue, K., Hong, P., Ma, C.: A lightweight dynamic pseudonym identity based authentication and key agreement protocol without verification tables for multiserver architecture. Arxiv preprint arXiv:1204.3831 (2012), http://arxiv.org/ abs/1204.3831

24. Kocher, P., Jafie, J., Jun, B.: Differential power analysis. In: Wiener, M.(ed.) CRYPTO 1999. LNCS, vol.1666, pp. 789-789. Springer, Heidelberg (1999)

25. Messerges, T.S., Dabbish, E.A., Sloan, R.H.: Examining smart-card security under the threat of power analysis attacks. IEEE Transactions on Computers 51(5), 541$552(2002)$

26. Kasper, T., Oswald, D., Paar, C.: Side-channel analysis of cryptographic rfids with analog demodulation. In: Juels, A., Paar, C. (eds.) RFIDSec 2012. LNCS, vol. 7055, pp. 61-77. Springer, Heidelberg (2012)

27. Florencio, D., Herley, C.: A large-scale study of web password habits. In: Proceedings of WWW 2007, pp. 657-666. ACM Press, New York (2007)

28. Klein, D.V.: Foiling the cracker: A survey of, and improvements to, password security. In: Proceedings of the 2nd USENIX Security Workshop. pp. 5-14 (1990)

29. Bao, F., Deng, R.: Privacy protection for transactions of digital goods. In: Qing, S., Okamoto, T., Zhou, J. (eds.) ICICS 2001. LNCS, vol. 2229, pp. 202-213. Springer, Heidelberg (2001)

30. Tang, C., Wu, D.: Mobile privacy in wireless networks-revisited. IEEE Transactions on Wireless Communications 7(3), 1035-1042 (2008)

31. Halevi, S., Krawczyk, H.: Public-key cryptography and password protocols. ACM Transactions on Information and System Security 2(3), 230-268 (1999) 